\title{
Advances in auditory display research
}

\author{
Brian F. G. Katz ${ }^{1}$ - Georgios Marentakis ${ }^{2}$
}

Received: 5 June 2016 / Accepted: 9 June 2016 / Published online: 20 June 2016

(C) SIP 2016

\begin{abstract}
The research community on Auditory Design has been active and organized now for almost 25 years. Work in Auditory Display is pluri-disciplinary/inter-disciplinary in nature, and involves disciplines such as perception, acoustics, digital signal processing, multimodality, ergonomics, aesthetics, cognition, etc. Published works have spanned fundamental, phenomenological, theoretical, applicative, and artistic studies. Over the years, various journals have had special issues related to such work. The range of the associated journals reflects the variety of implicated domains. This current special issue presents a collection of extended works that were selected from papers presented at the 2015 International Conference on Auditory Display. We present here an overview of the selection process and the accepted papers.
\end{abstract}

Keywords Auditory display - Sonification - Virtual reality . Auditory perception $\cdot$ Sound $\cdot$ Sonic interaction design

\section{Introduction}

The International Community on Auditory Display (ICAD) ${ }^{1}$ is a forum for presenting research on the use of sound to display data, monitor systems, and provide enhanced user interfaces for computers and virtual reality systems. It is unique in its singular focus on auditory displays and the

Brian F. G. Katz

brian.katz@limsi.fr

Georgios Marentakis

marentakis@iem.at

1 Audio Acoustics Group, LIMSI, CNRS, Université Paris-Saclay, Orsay, France

2 Institute of Electronic Music and Acoustics, Inffeldgasse 10/3, 8010 Graz, Austria array of perception, technology, and application areas that this encompasses. Research areas covered by ICAD include:

- Auditory exploration of data via Sonification (data controlled sound) and Audification (audible playback of data samples).

- Real-Time monitoring of multivariate data.

- Sound in Immersive Interfaces (Virtual Environments) and Teleoperation.

- Perceptual issues in Auditory Display.

- Sound in generalized computer interfaces.

- Technologies supporting Auditory Display creation.

- Data handling for Auditory Display systems.

- Applications of Auditory Display.

The field of Auditory Display has now a substantial history of almost 25 years. Although work on Auditory Display preceded the first International Conference on Auditory Display (ICAD) in 1992, the proceedings of which were published in [10], this conference has been widely acknowledged as the first effort to bring together researchers investigating the display of information using sound and the associated interaction techniques that are necessary. There are two subsequent milestones in the field that are also worth mentioning; the publication of the Sonification Handbook [8] and the collection of chapters relating to Sonic Interaction Design [5].

\section{Special issues}

Over the years, in addition to the aforementioned publications, eight special issues have been published that deal with topics directly relevant to auditory display. Three of

\footnotetext{
${ }^{1}$ http://www.icad.org/.
} 
them relate directly to the field of interactive sonification and have emerged out of the Interactive Sonification conferences $[2,4,7]$.

Two special issues were specifically entitled Auditory Display and comprised papers that were presented at, or originated in, ICAD conferences. Kramer and Walker [11] marked the 10th anniversary of the ICAD conference, while Stockman et al. [16] delivered an update on the research performed in this field. A more specialized issue has looked into the role of sound in motor perception and execution [13].

All of the special issues have acknowledged the importance of usability and very often studies in these issues included evaluations showcasing the extent to which the proposed methods can be applied in practice. A significant aspect that has recently begun receiving significant attention is the aesthetic one. In that direction [14], brought new insight into the field by considering aesthetic aspects of auditory display design. In many ways, this reflects a necessity fueled by developments in Human Computer Interaction research, which looks at display design beyond the information theoretic perspective.

\section{The ICAD 2015 conference}

The 21st International Conference on Auditory Display, with the theme "ICAD in Space", was held in Graz, Austria, in July of 2015. The conference was hosted by the Institute for Electronic Music and Acoustics of the University of Music and Performing Arts Graz with the support of the Technical University of Graz. The conference had both a scientific and an artistic track. In the scientific track, 56 articles, 25 extended abstracts, 12 student think tank proposals, and 9 workshop proposals were submitted. In addition, there were 59 artistic submissions (44 music and 15 sound installations). After a review process with area specific editors, 22 oral and 21 poster presentation were accepted. The music track was extremely competitive, with 8 music pieces and 5 sound installations being accepted.

\section{Paper selection process}

As part of the the Call for Papers, it was announced that a special issue of the Springer Journal on Multimodal User Interfaces would be dedicated to selected papers from the conference. Authors interested in candidature for this special journal issue were asked to indicate this during the submission process. Reviewers participating in the conference paper review process, were also asked to indicate whether a given submission had the potential for a journal publication. In total, the authors of 27 out of the 43 accepted papers indi- cated that they wished to be considered for selection in the special issue.

A Selection Committee was formed, comprising members of the ICAD 2015 Organisation Committee and the ICAD Board. During a meeting held at the end of the conference, the conference selection reviewers' ratings, as well as ratings by members of the Selection Committee were discussed. The committee identified 11 papers, based on quality and originality of the works, spanning various disciplines in the ICAD community. Authors of these papers were invited to submit extended versions of their conference papers, comprising at least $30 \%$ new material.

Consequently, submissions were subjected to the normal rigorous journal review process, that included blind peer reviews by at least two external reviewers, in addition to reviews by the two guest editors. At the conclusion of the review and revision process cycles, eight manuscripts were accepted to form this Special Issue on Auditory Display.

\section{Outlook}

The complex field of Auditory Display has been growing steadily over the past 2 decades. Research results in Auditory Display are increasingly often making their way into concrete applications and systems, which are readily available outside of laboratory conditions. With such advances, the rigour associated to auditory display research both fundamental and applied, as part of the interface design community, is also progressing, ensuring the scientific foundations necessary for this domain to continue to develop.

\section{Overview of the special issue contents}

The topics discussed in this special issue are central to auditory display research. They touch upon the use of sound to close the perception-action loop, the use of audio and multimodal displays in the communication of health risks to young people, multimodal interfaces for digital audio workstations, and spatial aspects related to auditory virtual environments.

Specifically, two articles on closing the perception-action loop are presented. Horsak et al. [9] presents and evaluates a wireless insole system that can be used to drive gait sonification while Stahl and Thoshkahna [15] presents and evaluates a sonification technique aimed to assist athletes in maintaining their heart rate within a pre-defined range. Tordini et al. [17] investigates auditory salience as an important design aspect of auditory displays, focusing specifically on the effect and interaction of tempo and brightness on salience between concurrent signals.

Walus et al. [18] presents a pilot study examining the use of auditory information to assist in the retention of information 
in the context of the communication of health risks related to alcohol consumption to young people. Metatla et al. [12] presents a participatory design approach applied to the design of audio-haptic interfaces for digital audio workstations for visually impaired sound engineers.

Finally, three articles deal with virtual audio display technology. Andreopoulou and Katz [1] discusses the use of subjective Head-Related Transfer Function (HRTF) evaluations for the creation of global similarity metrics of both HRTF data sets and the evaluation panel. Geronazzo et al. [6] discusses the simulation of interactive distance cues using a Digital Waveguide Mesh (DWM) acoustic synthesis model. Camier et al. [3] examines the extend to which simulation room reflections affect the perception of circular auditory motion with virtual sources.

\section{References}

1. Andreopoulou A, Katz BFG (2016) Subjective HRTF evaluations for obtaining global similarity metrics of assessors and assessees. J Multimodal User Interf. doi:10.1007/s12193-016-0214-y

2. Bresin R, Hermann T, Hunt A (2012) Interactive sonification. J Multimodal User Interf 5(3):85-86. doi:10.1007/ s12193-012-0095-7

3. Camier C, Boissinot J, Guastavino C (2016) On the robustness of upper limits for circular auditory motion perception. J Multimodal User Interf. doi:10.1007/s12193-016-0225-8

4. Degara N, Hunt A, Hermann T (2015) Interactive sonification [guest editors' introduction. IEEE MultiMedia 22(1):20-23. doi:10.1109/MMUL.2015.8

5. Franinović K, Serafin S (eds) (2013) Sonic interaction design. MIT Press, Cambridge

6. Geronazzo M, Avanzini F, Fontana F (2016) Auditory navigation with a tubular acoustic model for interactive distance cues and personalized head-related transfer functions. J Multimodal User Interfaces. doi:10.1007/s12193-016-0221-z
7. Hermann T, Hunt A (2005) Guest editors' introduction: An introduction to interactive sonification. IEEE MultiMedia 12(2):20-24. doi:10.1109/MMUL.2005.26

8. Hermann T, Hunt A, Neuhoff JG (2011) The sonification handbook. Logos Verlag, Berlin

9. Horsak B, Dlapka R, Iber M, Gorgas AM, Kiselka A, Gradl C, Siragy T, Doppler J (2016) Sonigait: a wireless instrumented insole device for real-time sonification of gait. J Multimodal User Interfaces. doi:10.1007/s12193-016-0216-9

10. Kramer G (ed) (1994) Auditory display: sonification, audification, and auditory interfaces. In: Proceedings of the Santa Fe institute studies in the science of complexity, vol XVIII. Addison-Wesley, Reading, MA

11. Kramer G, Walker BN (2005) Sound science: marking ten international conferences on auditory display. ACM Trans Appl Perception (TAP) 2(4):383-388. doi:10.1145/1101530.1101531

12. Metatla O, Martin F, Parkinson A, Bryan-Kinns N, Stockman T, Tanaka A (2016) Audio-haptic interfaces for digital audio workstations. J Multimodal User Interfaces. doi:10.1007/ s12193-016-0217-8

13. Murgia M, Galmonte A (2015) The role of sound in motor perception and execution. Open Psychol J 8(3):171-173. doi:10.2174/ 1874350101508010171

14. Schedel M, Worrall D (2014) Editorial. Organized sound. Open Psychol J 19:1-3. doi:10.1017/S1355771813000356

15. Stahl B, Thoshkahna B (2016) Design and evaluation of the effectiveness of a sonification technique for real time heart-rate data. J Multimodal User Interfaces. doi:10.1007/s12193-016-0218-7

16. Stockman T, Roginska A, Walker B, Metatla O (2012) Guest editors' note: special issue on auditory display. J Audio Eng Soc 60(7/8):1. http://www.aes.org/journal/online/JAES_V60/7_8/

17. Tordini F, Bregman AS, Cooperstock JR (2016) Prioritizing foreground selection of natural chirp sounds by tempo and spectral centroid. J Multimodal User Interfaces. doi:10.1007/ s12193-016-0223-x

18. Walus BP, Pauletto S, Mason-Jones A (2016) Sonification and music as support to the communication of alcohol-related health risks to young people. J Multimodal User Interf. doi:10.1007/ s12193-016-0220-0 